\title{
Satisfação e sobrecarga de trabalho da equipe de enfermagem em um hospital psiquiátrico
}

\author{
Satisfaction and workload of the nursing team in a psychiatric hospital
}

La satisfacción y la sobrecarga laboral del equipo de enfermería en un hospital psiquiátrico

Wanderson de Carvalho Menezes ${ }^{1 *}$, Cláudio Alves de Melo², Fabiana Pereira Passos ${ }^{2}$, Rodrigo da Silva Almeida ${ }^{2}$.

\section{RESUMO}

Objetivo: Identificar os níveis de satisfação e sobrecarga relacionados ao trabalho da equipe de enfermagem de um hospital psiquiátrico. Métodos: Estudo exploratório descritivo, quantitativo, do tipo transversal, realizado em 2020, na cidade de Brasília, junto aos profissionais de enfermagem. A população foi desenhada dos 130 profissionais que atuam na internação, sendo a amostra de 50 participantes. Foram utilizados para coleta de dados: Questionário contendo dados sociodemográficos e escalas Brasileiras de Avaliação da Satisfação (Satis-BR abreviada) e da sobrecarga (Impacto-BR abreviada) ambas para serviços de saúde mental. Resultados: Verificou-se que mais da metade $66 \%$ eram do sexo feminino, $40 \%$ estavam na faixa de 45-54 anos de idade, $54 \%$ vivem com companheiro e grande maioria $82 \%$ eram compostas por técnicos e/ou auxiliares de enfermagem. O escore de satisfação da equipe ficou em 3,22 indicando moderado nível de satisfação, já a sobrecarga foi de 2,11 apontando moderado impacto no trabalho. Conclusão: $O$ estudo demonstrou graus moderados de satisfação e sobrecarga de trabalho, tal resultado, mostra a necessidade de melhoria das condições de trabalho por meio da revisão dos projetos institucionais, uma vez que esses índices refletem diretamente na assistência prestada por esses trabalhadores.

Palavras-chave: Carga de trabalho, Equipe de enfermagem, Saúde mental, Saúde do trabalhador, Satisfação no emprego.

\begin{abstract}
Objective: Identify the levels of satisfaction and workload related to the work of the nursing team in a psychiatric hospital. Methods: Descriptive, quantitative, cross-sectional exploratory study, carried out in 2020, in the city of Brasília, with nursing professionals. The population was drawn from the 130 professionals assigned to the hospital, with a sample of 50 participants. A questionnaire containing sociodemographic data and Brazilian scales of Satisfaction Assessment (abbreviated Satis-BR) and overload (abbreviated BR-impact) were used for data collection, both for mental health services. Results: It was found that more than half $66 \%$ were female, $40 \%$ were aged $45-54$ years old, $54 \%$ lived with a partner and the vast majority $82 \%$ were technicians and / or nursing assistants. The satisfaction score of the team was 3.22 , indicating a moderate level of satisfaction, as for workload it was 2.11, indicating moderate impact at work. Conclusion: The study demonstrated moderate degrees of satisfaction and work overload, this result, showing the need to improve working conditions through the review of institutional projects, since these indexes directly reflect on the care provided by these workers.
\end{abstract}

Key words: Workload, Nursing team, Mental health, Occupational health, Job satisfaction.

\footnotetext{
${ }^{1}$ Escola Superior de Ciências da Saúde (ESCS) / Fundação de Ensino e Pesquisa em Ciências da Saúde (FEPECS), Brasília - DF. *E-mail: wenzo10@live.com

2 Secretaria de Estado de Saúde do Distrito Federal (SES-DF), Brasília - DF.
}

PUBLICADO EM: 5/2021 


\section{RESUMEN}

Objetivo: Identificar los niveles de satisfacción y sobrecarga relacionados con el trabajo del equipo de enfermería en un hospital psiquiátrico. Métodos: Estudio exploratorio descriptivo, cuantitativo, transversal, realizado en 2020, en la ciudad de Brasilia, con profesionales de enfermería. La población se extrajo de los 130 profesionales adscritos al hospital, con una muestra de 50 participantes. Se utilizaron para la recopilación de datos: cuestionario que contiene datos sociodemográficoy las escalas brasileñas de Evaluación de la Satisfacción (abreviado Satis-BR) y sobrecarga (abreviado BR-impacto), ambas para los servicios de salud mental. Resultados: Se encontró que más de la mitad $66 \%$ eran mujeres, $40 \%$ tenían entre 45 y 54 años, $54 \%$ vivían en pareja y la gran mayoría $82 \%$ eran técnicos y / o auxiliares de enfermería. La puntuación de satisfacción del equipo fue 3.22, lo que indica un nivel moderado de satisfacción, la carga de trabajo fue 2,11, lo que indica moderado impacto en el trabajo. Conclusión: El estudio demostró grados moderados de satisfacción y sobrecarga laboral, este resultado, mostrando la necesidad de mejorar las condiciones de trabajo a través de la revisión de proyectos institucionales, ya que estos índices reflejan directamente la atención brindada por estos trabajadores.

Palabras clave: Carga de trabajo, Equipo de enfermería, Salud mental, Salud laboral, Satisfacción laboral.

\section{INTRODUÇÃO}

O movimento da Reforma Psiquiátrica Brasileira trouxe intensas transformações nas últimas três décadas para assistência às pessoas que necessitam de tratamento mental. Iniciada com o processo de redemocratização, que fundamentou na indignação quanto às desigualdades e a mercantilização da saúde e da loucura (CAFÉ LA, et al., 2020; SAMPAIO ML e BISPO JÚNIOR JP, 2021).

Tal mudança, fez com que a Organização Mundial da Saúde (OMS), recomendasse avaliações contínuas dos estabelecimentos de saúde mental, sobre a ótica dos três agentes: pacientes, familiares e profissionais. Essa recomendação visa a constante melhoria dos indicadores dos serviços, através do diagnóstico das dificuldades no tratamento com objetivo de fornecer condições para sua resolução, assim como, evitar o retrocesso ao modelo de tratamento pautado na exclusão social que só cronifica e exclui o sujeito de seu corpo social (LEAL RMAC, et al., 2012; TREVISAN E, et al., 2019).

Porém, com a publicação da Nota Técnica n 11/2019-CGMAD/DAPES/SAS/MS, fruto de portarias, resoluções e editais que aponta e redireciona para a "Nova Política Nacional de Saúde Mental", observa-se novamente a inserção dos hospitais psiquiátricos na Rede de Atenção Psicossocial (RAPS). Ocorre também, uma mudança de discurso na política, ao afirmar que os serviços da rede devem ser harmônicos e complementares entre si, não mais considerando os serviços como sendo substitutivos um do outro (BRASIL, 2019; CRUZ NFO, et al., 2020).

Quando analisamos o arcabouço da legislação do Sistema Único de Saúde (SUS), tal direcionamento segue na contramão da Lei da Reforma Psiquiátrica (Lei n. 10216 de 2001) e de todo um processo de mobilização histórico social e política na luta pela garantia de direitos e tratamento em serviços extrahospitalares, onde a cultura e a linguagem do sujeito e livre na expressão de sua subjetividade (BRASIL, 2001; SAMPAIO ML e BISPO JÚNIOR JP, 2021).

E nesse contexto político e social, que a enfermagem em saúde mental se encontra. É importante salientar que só a partir da Resolução COFEN n. 599/2018 que a equipe de enfermagem passa a ter uma norma técnica que regulamenta a atuação em Saúde Mental e Psiquiatria. Em estudo de revisão narrativa para compreender a enfermagem em saúde mental, Café LA, et al. (2020) diz que a atuação da equipe de enfermagem está pautada na Sistematização da Assistência de Enfermagem (SAE), assim como nos preceitos da Reforma Sanitária e Reforma Psiquiátrica, sendo estes profissionais de fundamental importância para efetivação dessas políticas.

A enfermagem por estar em constante contato com o sofrimento e dor das pessoas que cuidam, mantendo interações com ambiente, equipe e paciente, configurando-se uma tríade cheia de riscos ocupacionais 
relacionados com esgotamento profissional, desgaste psíquico, sobrecarga de trabalho, indolência e culpa, bem como a insegurança de assistir pacientes em crise psicótica. Esses fatores interferem diretamente na assistência prestada por esses profissionais (MEDEIROS ARS, et al., 2019).

Dessa forma, estudos demonstram que os profissionais de enfermagem estão predispostos a desencadearem eventos estressores contínuos ao ponto de serem acometidos pela síndrome de Burnout, principalmente quando estes prestam assistência direta aos pacientes internados (SILVA CC, et al., 2020; GUTIERREZ KPM, et al., 2020).

Tem-se ainda, as condições e dinâmica de trabalho da atenção à saúde mental, que contribui diretamente ao risco do adoecimento desses profissionais, como: repetitividade de tarefa, estrutura física inadequada e com barulho, bem como, da possibilidade de serem agredidos ao final da jornada de trabalho (SOUSA KHJF, et al., 2018). Situações que influenciam diretamente na sobrecarga e satisfação destes trabalhadores (VIEIRA GLC, 2017).

Diante do exposto, o presente estudo teve como objetivo identificar os níveis de satisfação e sobrecarga relacionados ao trabalho da equipe de enfermagem em um hospital psiquiátrico do Distrito Federal.

\section{MÉTODOS}

Trata-se de estudo transversal e quantitativo, utilizando amostra não probabilística e por conveniência de 50 trabalhadores de enfermagem de ambos os sexos, que exerciam atividades assistenciais em um hospital que é referência no atendimento de emergência a pacientes com transtornos mentais, localizado na região administrativa de Taguatinga, Distrito Federal. A coleta sucedeu-se nos meses de outubro e novembro de 2020, em etapas únicas, por meio de questionário autoadministrado. Na ocasião os sujeitos da pesquisa foram convidados a participarem em seu próprio ambiente de trabalho no período da manhã, tarde e noite.

O critério de inclusão foi fazer parte da equipe de enfermagem que atua diretamente na assistência psiquiátrica, enquanto os de exclusão foram estar gozando de férias e licenças ou estar afastados das funções assistenciais no período da coleta de dados.

Os profissionais foram convidados a responder o questionário sociodemográfico e escalas Brasileiras de Avaliação da Satisfação (Satis-BR abreviada) e da sobrecarga (Impacto-BR abreviada) dos profissionais de saúde mental. Essas escalas foram elaboradas pela divisão de saúde mental da World Health Organization (WHO, 1996) e validadas no Brasil (BANDEIRA M, 2007). O questionário sociodemográfico abordou os seguintes dados: estado civil, sexo, tempo de vínculo com a instituição, tempo de experiência na saúde mental, carga horária de trabalho, turno de trabalho e percepção de segurança no ambiente de trabalho.

A escala SATIS-BR avalia o grau de satisfação da equipe em relação aos serviços de saúde mental em que trabalham. As respostas estão dispostas em escala ordinal (tipo Likert) de cinco pontos, que vai de "Muito insatisfeito" (1) a "Muito satisfeito" (5), agrupadas em quatro (subescalas ou fatores). O primeiro fator avalia o grau de satisfação da equipe com relação à qualidade dos serviços oferecidos aos pacientes (alfa de Cronbach $=0,83$ ), o segundo fator avalia o grau de satisfação da equipe com relação à sua participação no serviço (alfa de Cronbach $=0,72$ ), o terceiro fator avalia o grau de satisfação da equipe em relação às condições gerais de trabalho (alfa de Cronbach $=0,77$ ) e o quarto fator avalia o grau de satisfação da equipe a respeito do seu relacionamento no serviço (alfa de Cronbach $=0,63$ ). A escala global com os 32 itens possui uma consistência interna de alfa de Cronbach de 0,89 (BANDEIRA M, et al., 2000; BANDEIRA M, et al., 2007)

Enquanto a escala IMPACTO-BR tem como finalidade avaliar a sobrecarga sentida pelos profissionais de saúde mental, em consequência do trabalho com pessoas em sofrimento mental. As respostas estão dispostas em escala ordinal (tipo Likert) de cinco pontos, que varia conforme a pergunta em "De forma alguma" e "Nunca" (1) a "Extremamente" a "Sempre" (5), agrupadas em três (subescalas ou fatores). O primeiro fator avalia o grau de sobrecarga referente aos efeitos ressentidos pela equipe na sua saúde física e mental (alfa de Cronbach $=0,78$ ), o segundo fator avalia o grau da sobrecarga de trabalho no funcionamento da equipe (alfa de Cronbach $=0,77$ ), e o terceiro fator busca avaliar o grau de sobrecarga referente às repercussões 
emocionais do trabalho (alfa de Cronbach $=0,70$ ). A escala global com os 18 itens também apresenta uma elevada consistência interna alfa de Cronbach de 0,87 (BANDEIRA M, et al., 2000; BANDEIRA M, et al., 2007).

A análise estatística utilizou do software Statistical Package for the Social Sciences (SPSS) 20.0 para Windows. Para analisar os itens da escala Likert foi utilizado o cálculo do Ranking Médio (RM) proposto por Oliveira LH (2005). Na análise descritiva dos dados, foi realizado o cálculo das frequências e médias ponderadas das escalas e seus respectivos fatores para o cálculo do Ranking Médio.

A pesquisa foi aprovada pelo Comitê de Ética em Pesquisa, sob número CAAE 31976720.6.0000.5056 e parecer número 4.274.607, após aquiescência do local onde se deu a coleta de dados. Todos os participantes do estudo leram, assinaram e receberam o Termo de Consentimento Livre e Esclarecido.

\section{RESULTADOS E DISCUSSÃO}

O fator limitante do estudo está atrelado a avaliação apenas da equipe de enfermagem, a ampliação do estudo com a equipe multiprofissional de saúde mental e com número maior de participantes para que haja uma leitura mais ampla do hospital psiquiátrico faz-se necessário. Outro fator, concentra na utilização apenas dos instrumentos de avaliação de sobrecarga e satisfação da equipe em serviços de saúde mental. Portanto, para melhorar investigações futuras faz-se necessário correlacioná-las as variáveis voltadas às questões ocupacionais dentre outras questões que possam avaliar esses profissionais que atuam na assistência em saúde mental.

Dos 130 profissionais de enfermagem lotados nas enfermarias de internação e pronto socorro psiquiátrico, apenas 50 aceitaram participar da pesquisa, os demais, encontravam-se de férias, licenças ou não estavam escalados nas semanas em que ocorreram as coletas de dados.

Quanto a caracterização do estudo, verificou-se que mais da metade $66 \%$ eram do sexo feminino, $40 \%$ estavam de $45-54$ anos de idade, $54 \%$ vivem com companheiro (a) e grande maioria $82 \%$ era composta por técnicos e/ou auxiliares de enfermagem, sendo que $56 \%$ dos participantes possuem ensino superior e $56 \%$ exerciam carga horaria de 40 horas semanais. Ao ser perguntado quanto à percepção de segurança do hospital psiquiátrico, a maioria $64 \%$ responderam que se sentem sim seguras. Os resultados demonstram ainda que 58\% realizam suas atividades no período diurno e 52\% possuem experiência de 1-10 anos na área de saúde mental (Tabela 1).

Diante dos dados apresentados verifica-se que a variável sexo feminino apresentou-se elevada em comparação ao sexo masculino respectivamente $66 \%$ e $34 \%$. De igual modo, autores de estudo congênere a este, desenvolvidos em hospitais psiquiátricos do Paraná e Minas Gerais também identificaram predomínio de mulheres $80,9 \%$ e $72,2 \%$ entre os sujeitos (ALVES SR, et al., 2017; VIEIRA GLC, 2017).

Observa-se que as mulheres já de muitas décadas têm na enfermagem um papel destaque nos serviços de saúde do Brasil, assim como seu papel para a feminilização da saúde brasileira. Entretanto, é importante pontuar que há um processo de masculinização de 13,4\%, o que configura uma nova tendência do crescimento do contingente masculino na categoria (BRASIL, 2017). 
Tabela 1 - Características sociodemográficas e ocupacionais dos profissionais de enfermagem do Hospital, $\mathrm{n}=50$.

\begin{tabular}{|c|c|c|}
\hline Variável & $\mathbf{N}$ & $\%$ \\
\hline \multicolumn{3}{|l|}{ Sexo } \\
\hline Masculino & 17 & 34 \\
\hline Feminino & 33 & 66 \\
\hline \multicolumn{3}{|l|}{ Idade } \\
\hline $25-34$ & 9 & 18 \\
\hline $35-44$ & 16 & 32 \\
\hline $45-54$ & 20 & 40 \\
\hline $55-64$ & 5 & 10 \\
\hline \multicolumn{3}{|l|}{ Escolaridade } \\
\hline Ensino médio técnico & 17 & 34 \\
\hline Superior & 28 & 56 \\
\hline Pós-graduação & 5 & 10 \\
\hline \multicolumn{3}{|l|}{ Estado civil } \\
\hline Com companheiro (a) & 27 & 54 \\
\hline Sem companheiro (a) & 23 & 46 \\
\hline \multicolumn{3}{|l|}{ Cargo } \\
\hline Auxiliar e técnico de enfermagem & 41 & 82 \\
\hline Enfermeiro & 9 & 18 \\
\hline \multicolumn{3}{|l|}{ Carga horária semanal } \\
\hline 20 & 20 & 40 \\
\hline 40 & 28 & 56 \\
\hline$>40$ & 2 & 4 \\
\hline \multicolumn{3}{|l|}{ Turno de trabalho } \\
\hline Diurno & 29 & 58 \\
\hline Noturno & 5 & 10 \\
\hline Diurno e noturno & 16 & 32 \\
\hline \multicolumn{3}{|l|}{ Experiência na saúde mental em anos } \\
\hline $1-10$ & 26 & 52 \\
\hline $11-20$ & 24 & 34 \\
\hline$>20$ & 7 & 14 \\
\hline \multicolumn{3}{|l|}{ Percepcão de segurança } \\
\hline Sim & 32 & 64 \\
\hline Não & 18 & 36 \\
\hline
\end{tabular}

Fonte: Menezes WC, et al., 2021.

Ainda em relação a caracterização dos participantes, constatou-se que a grande maioria $82 \%$ eram técnicos e/ou auxiliares de enfermagem. O que vai de encontro com Pesquisa Perfil da Enfermagem Brasileira, onde afirma que $77 \%$ da enfermagem brasileira é composta por esses profissionais (BRASIL, 2017). Outra estatística relevante, é que $56 \%$ possuem ensino superior, destes $48 \%$ são de técnicos e/ou auxiliares de enfermagem. Para Frota MA, et al. (2020) o desejo de se tornar um profissional competitivo e de ascender socialmente mediante a conclusão do ensino superior é um dos fatores que levam esses profissionais de nível médio a se graduarem.

Sobre a percepção positiva de segurança dos participantes. Estudo realizado por Vieira GLC, (2017) em dois hospitais psiquiátricos de Belo Horizonte, diverge dessa amostra ao demonstrar que a maioria dos profissionais de enfermagem afirmaram não se sentirem seguros nesses espaços. Dessa forma, verifica-se que mesmo ocorrendo divergência com a literatura científica, os serviços devem se organizar com intuito de trabalhar ações de prevenção, qualificação e estratégias coletivas para mitigar qualquer tipo de violência em seus espaços.

Os dados obtidos, através da Escala de Avaliação SATIS-BR, permitiram calcular os escores de satisfação geral e por fatores da escala e sujeito para o conjunto da amostra. O escore global de satisfação foi de 3,22 com variação de 1,28 a 4,8 valor situado entre "indiferente" (3) e "satisfeito" (4) na escala Likert (Tabela 2). 
Tabela 2 - Ranking médio dos fatores relacionados à satisfação dos profissionais de enfermagem do Hospital, $\mathrm{n}=50$.

\begin{tabular}{lccc}
\hline \multirow{2}{*}{ Fatores da escala SANTIS-BR } & \multicolumn{3}{c}{ Ranking médio } \\
\cline { 2 - 4 } & - & Mínimo & Máximo \\
\hline $\begin{array}{l}\text { Satisfação global } \\
\quad \text { Fator 1: Satisfação da equipe em relação a qualidade }\end{array}$ & 3,22 & 1,28 & 4,88 \\
dos serviços oferecidos & 1 & 4,44 \\
$\quad$ Fator 2: Satisfação da equipe em relação a & 2,88 & 1 & 4,86 \\
$\begin{array}{l}\text { participação no serviço } \\
\quad \text { Fator 3: Satisfação da equipe em relação às condições }\end{array}$ & 3,16 & 1,22 & 4,78 \\
$\begin{array}{l}\text { de trabalho } \\
\quad \text { Fator 4: Satisfação da equipe em relação ao }\end{array}$ & 3,69 & 1,33 & 5 \\
relacionamento no serviço & & & \\
\hline
\end{tabular}

Fonte: Menezes WC, et al., 2021.

Portanto, revelam que os profissionais estudados apresentaram níveis moderados de satisfação, situados entre a indiferença e satisfação". Estes resultados assinalam a necessidade de rever os projetos organizacionais, para que o nível de satisfação aumente e consequentemente a qualidade da assistência prestada no serviço.

$\mathrm{Na}$ avaliação dos fatores, o que teve maior escore de satisfação foi o Fator 4 - relacionamento no serviço 3,69 com variação de 1,3 a 3-5. As demais, foram Fator 1 - satisfação da equipe em relação a qualidade dos serviços oferecidos 3,35 e Fator 3 - satisfação da equipe em relação às condições de trabalho 3,16. Enquanto a menor satisfação 2,88 ficou com o fator que avalia a participação no serviço.

Observou-se que as três questões com maiores escores de satisfação foram a competência profissional da equipe do serviço 3,92 a responsabilidade que os profissionais têm no serviço 3,76 e clima no ambiente de trabalho 3,72. Por outro lado, a participação nos processos de tomada de decisão no serviço 2,56 a atenção dada às suas opiniões 2,66 e conforto e aparência do serviço 2,72 foram destaques negativos nos escores de satisfação.

Dessa forma, observa-se que os níveis de insatisfação da equipe de enfermagem estão diretamente relacionados aos níveis de participação que esses profissionais têm no serviço. Quando olhamos para os níveis de satisfação, predominou o relacionamento gerado pelo bom ambiente de trabalho.

Estudos recentes demonstram que profissionais que têm apoio social no ambiente de trabalho demonstram maiores níveis de satisfação (ASSUNCAO AÁ e PIMENTA AM, 2020). Além disso, fatores ligados ao relacionamento no trabalho são, em geral, indicadores positivos de satisfação por profissionais da saúde mental em outros estudos (DIAS GC e FUREGATO ARF, 2016; VIEIRA GLC, 2017; TREVISAN E, et al., 2019).

É importante que os gestores deem visibilidade às experiências dos trabalhadores para incluí-los na tomada de decisão. Qualificando os processos de trabalho através da capacidade de analisar essa relação, produzindo mudanças nos modelos de gerir o cuidado em saúde das instituições.

Existe também, a capacidade de adaptação aos momentos geradores de estresse na área da psiquiatria. Estudo realizado em Cingapura com enfermeiros psiquiátricos, demonstrou que o grau de satisfação está positivamente ligado aos níveis de resiliência (ZHENG Z, et al., 2017). Percebe-se, assim, que tal habilidade psicológica faz-se necessária para enfrentar as adversidades que existem na assistência às pessoas com sofrimento mental, principalmente em ambiente hospitalar.

Ferreira AP, (2015), em investigação realizada num Centro de Atenção Psicossocial com profissionais de saúde, concluiu que o estresse estava diretamente ligado aos índices de satisfação. Ou seja, menores índices de satisfação maiores índices de estresse no trabalho.

Portanto, fica evidente que as instituições hospitalares necessitam de um replanejamento, por meio de ações ou estratégias futuras, que sejam direcionadas para melhorias nas condições e processo de trabalho, 
para que dessa forma, o ambiente do trabalho possa ser fonte de satisfação e benefício. Para assim, alcançar os objetivos estabelecidos nas políticas de saúde, da cultura de segurança e da cultura organizacional.

A Tabela 3 traz os dados obtidos através da Escala de Avaliação IMPACTO-BR, verificou-se que o escore de sobrecarga global dos profissionais estudados foi de 2,11 com variação de 1 a 4,44. Encontrando-se entre "De forma alguma" é "Mais ou menos" ou "Nunca" e "Mais ou menos" (2 a 3) na Likert. Assim como seus três fatores que foram avaliados através do RM (Tabela 3).

Tabela 3 - Ranking médio dos fatores relacionados à sobrecarga de trabalho dos profissionais de enfermagem do Hospital, $\mathrm{n}=50$.

\begin{tabular}{lccc}
\hline \multirow{2}{*}{ Fatores da escala IMPACTO-BR } & \multicolumn{3}{c}{ Ranking Médio } \\
\cline { 2 - 4 } & - & Mínimo & Máximo \\
\hline Sobrecarga global & 2,11 & 1 & 4,44 \\
Fator 1: Impacto sobre a saúde física e mental & 1,91 & 1 & 4,6 \\
Fator 2: Impacto sobre o funcionamento da equipe & 2,44 & 1 & 5 \\
Fator 3: Impacto emocional do trabalho & 2,21 & 1 & 4,2 \\
\hline
\end{tabular}

Fonte: Menezes WC, et al., 2021.

Apesar do hospital psiquiátrico possuir algumas características que desencadeiam sobrecarga nos profissionais de saúde principalmente da equipe de enfermagem. Nessa instituição não foi encontrado escores altos de sobrecarga para esses profissionais. Além disso, investigações como essa em instituições psiquiátricas trazem níveis ainda mais baixos de sobrecarga (DIAS GC e FUREGATO ARF, 2016; ALVES SR, et al., 2016).

Em revisão Muniz DC, et al. (2019) demonstra que a sobrecarga de trabalho traz consequências como: depressão, estresse, tensão, mau ambiente de trabalho entre outras condições psicológicas e fatores que influenciam direta ou indiretamente para o adoecimento do profissional de saúde.

Os valores encontrados demonstram uma certa proximidade do grau de sobrecarga aceitável, mas ainda assim os profissionais de enfermagem percebem níveis moderados de sobrecarga no ambiente onde atuam. Portanto, melhorar as condições de trabalho para esses profissionais e melhorar a qualidade da assistência prestada no serviço.

O fator com maior grau de sobrecarga foi o relacionado ao impacto sobre o funcionamento da equipe 2,44 com variação de 1 a 5 . Já os menores respectivamente foram: Fator 1 impacto sobre a saúde física e mental 1,91 e Fator 3 ligado ao impacto emocional do trabalho 2,21.

As variáveis individuais com maiores escores de sobrecarga destaca-se, o receio da possibilidade de ser fisicamente agredido por um paciente 3,78. O sentimento de cansaço quando termina a jornada de trabalho 2,68 e sentimento de frustração no resultado de seu trabalho com os portadores de transtorno mental 2,62.

Enquanto o fato de ter contato com pessoas portadores de transtorno mental, e se isso está afetando a vida pessoal do profissional 1,32 foi a variável com menor impacto de sobrecarga, entre as três menores, seguida pela estabilidade emocional pelo contato com portadores de transtorno mental 1,38 . $O$ fato de se sentir estressado por causa do trabalho em saúde mental 1,38 , foi a terceira variável com menor grau de sobrecarga.

A história da loucura, expõe a visão que o louco é perigoso. Somado a isso, os problemas de moralidade e fenômenos que animalizam o próprio homem com sua percepção de periculosidade e ser nocivo à sociedade (OLIVEIRA FV, 2020). Mas, quem é esse "louco"? Ao analisarmos o dicionário eletrônico Houaiss (2009) identificamos uma origem obscura, histórica e controversa, definido como "aquele cujo comportamento ou raciocínio denota alterações patológicas das faculdades mentais".

Providello GGD e Yasui S (2013) ressalta que entender as diferentes sensibilidades em relação à loucura é um passo importante para compreender os estereótipos da loucura, que foi construído ao longo da história pelo próprio homem a partir de tecnologias dos saberes sobre esse corpo específico. Para Foucault (2006, p. 
156) a loucura nada mais é que a ausência de obra, ou seja, uma condição que leva o sujeito ao silêncio de sua própria linguagem subjetiva.

$\mathrm{Na}$ idade clássica, a loucura se tornou uma linguagem interdita em hospitais gerais, assim como qualquer linguagem que ia contra o domínio da verdade ou eram colocados na prateleira da desrazão. Com a libertação dos loucos no século XIX, essas pessoas passaram da exclusão social, moral e jurídica para uma questão médica de exclusão que é exercida pelo poder da doença mental (PROVIDELLO GGD e YASUI S, 2013).

Portanto, para os profissionais de enfermagem o entendimento desse contexto histórico da loucura faz-se necessário para que possamos construir espaços de cuidado em saúde mental menos excludente e mais centrado no cuidado humano das pessoas que estão em sofrimento mental.

Estudo realizado no interior paulista caracterizou os riscos psicossociais no trabalho de enfermeiros que trabalham em um hospital psiquiátrico de médio porte. A investigação concluiu, que os riscos se relacionam a formação do profissional, relacionamento interpessoal, sobrecarga de trabalho, falta de recursos humanos de enfermagem, equipamento de trabalho e interface do trabalho-família e ambiente violento (SCOZZAFAVE MCS, et al. 2019).

A presença desses riscos incorre em consequências para esses trabalhadores que se sentem cansados, esgotados, e por vezes, estressados pela complexidade do trabalho em um hospital psiquiátrico. Oliveira RM, et al. (2019) aponta em estudo, que os profissionais de enfermagem sentem mais sobrecarregados emocionalmente cuidados dos pacientes psiquiátricos que em outras especialidades de um hospital geral. Ademais, as violências psicológicas e institucionais interferem significativamente na percepção da equipe de enfermagem, causando sofrimento e perda de qualidade na prática do cuidado ao paciente (PAULA GS, et al., 2017).

Santos CSCS, et al, (2020) avalia que as instituições hospitalares devem promover condições de trabalho, com recursos físicos e humanos. Trazendo coerência aos processos organizacionais do trabalho, apesar de existir a implementação de políticas de saúde do trabalhador, os serviços devem constantemente avaliar os níveis de sobrecarga de seus colaboradores, para que a segurança do paciente e na qualidade da assistência de enfermagem.

\section{CONCLUSÃO}

Os resultados mostraram que os profissionais da equipe de enfermagem apresentam moderado grau de satisfação, sentem-se mais satisfeitos com os relacionamentos no ambiente de trabalho e com a qualidade dos serviços oferecidos. Moderada sobrecarga de trabalho, mas bem próximo aos índices de menor impacto, esse índice aumenta com a percepção negativa ao funcionamento da equipe. A maior insatisfação deu-se pelo fato que a equipe não se ver integrada nos processos do serviço, enquanto a menor sobrecarga de impacto ficou com a percepção negativa sobre a saúde física e mental. Em vista desses resultados, percebese a necessidade da revisão dos projetos institucionais, na busca de melhorias das condições de trabalho, sabendo que a satisfação está diretamente ligada aos fatores de impacto no trabalho. A melhoria desses índices também irá refletir na qualidade da assistência prestada por esses trabalhadores.

\section{REFERÊNCIAS}

1. ALVES SR, et al. Satisfação da equipe de enfermagem em serviços de saúde mental: um estudo comparativo entre profissionais de instituição pública e privada. REME - Rev Min Enferm., 2017; 21: e-993.

2. ALVES SR, et al. Sobrecarga de trabalho da Enfermagem em saúde mental. Rev Rene. 2016; 17(5): 684-90.

3. ASSUNCAO AÁ, PIMENTA AM. Satisfação no trabalho do pessoal de enfermagem na rede pública de saúde em uma capital brasileira. Ciênc. Saúde Coletiva, 2020; 25(1): 169-180.

4. BANDEIRA M, et al. Escalas brasileiras de avaliação da satisfação (SATIS-BR) e da sobrecarga (IMPACTO-BR) da equipe técnica em serviços de saúde mental. J Bras Psiquiatria, 2000; 49(4): 145-152.

5. BANDEIRA, M. et al. Satisfação e sobrecarga de profissionais de saúde mental: Validade de construto das escalas SATIS-BR e IMPACTO-BR. J Bras de Psiquiatria, 2007; 56(4): 280-286. 
6. BRASIL. Ministério da Saúde. Lei n. 10.216, de 6 de abril de 2001. Dispõe sobre a proteção e os direitos das pessoas portadoras de transtornos mentais e redireciona o modelo assistencial em saúde mental. Diário Oficial da União, $2001 ; 1$.

7. BRASIL. Ministério da Saúde. Secretaria de Atenção à Saúde. Departamento de Ações Pragmáticas Estratégicas. Coordenação-Geral de Saúde Mental, Álcool e Outras Drogas. Nota Técnica n. 11/ 2019, de 4 de fevereiro de 2019.

8. BRASIL. Perfil da enfermagem no Brasil: Relatório Final. Conselho Federal de Enfermagem / Fundação Oswaldo Cruz. Rio de Janeiro, 2017.

9. CAFÉ LA, et al. A atuação do enfermeiro na saúde mental. Revista Artigos. Com, 8 out. 2020; 21: e5016.

10. Conselho Federal de Enfermagem (COFEN). Resolução Cofen no 599/2018. Norma técnica para atuação da equipe de Enfermagem em Saúde Mental e Psiquiatria, 2018.

11. CRUZ NFO, et al. Retrocesso da reforma psiquiátrica: o desmonte da política nacional de saúde mental brasileira de 2016 a 2019. Trab. Educ. Saúde, 2020; 18(3): e00285117.

12. DIAS GC, FUREGATO ARF. Impacto do trabalho e satisfação da equipe multiprofissional de um hospital psiquiátrico. Revista Enfermagem UERJ, [S.I.], jul. 2016; 24(1): e8164.

13. FERREIRA AP. Satisfaction, workload and stress among providers of a mental health service. Rev Bras Med Trab. 2015; 13(2): 91-99.

14. FOUCAULT M. Loucura, literatura, sociedade. In: Motta, Manoel Barbosa (Org.). Problematização do sujeito: psicologia, psiquiatria e psicanálise. Rio de Janeiro: Forense Universitária, 2006; p.232-258.

15. GUTIERREZ KPM, et al. Burnout Syndrome in Nursing Professionals in Punta Arenas, Chile. Texto contexto - enferm., 2020; 29(spe): e20190273.

16. HOUAISS. Dicionário eletrônico Houaiss da língua portuguesa. Rio de Janeiro: Objetiva, 2009.

17. LEAL RMAC, et al. Avaliação da qualidade de um serviço de saúde mental na perspectiva do trabalhador: satisfação, sobrecarga e condições de trabalho dos profissionais. Psicol Teor Prática, 2012; 14(1): 15-25.

18. MEDEIROS ARS, et al. O burnout em profissionais de enfermagem que atuam em um complexo psiquiátrico. Rev. Enferm. UFSM - REUFSM, 2019, 9: e36, 1-16.

19. MUNIZ DC, et al. A saúde do enfermeiro com sobrecarga de trabalho. Rev Inic Cient Ext., 2019; 2(Esp.2): 274-9.

20. OLIVEIRA JF, et al. Satisfação profissional e sobrecarga de trabalho de enfermeiros da área de saúde mental. Ciênc. Saúde coletiva, 2019; 24(7): 2593-2599.

21. OLIVEIRA LH. Exemplo de cálculo de Ranking Médio para Likert. Notas de Aula. Metodologia Científica e Técnicas de Pesquisa em Administração. Mestrado em Adm. e Desenvolvimento Organizacional. PPGA CNEC/FACECA: Varginha, 2005.

22. OLIVEIRA RM, et al. Cuidados de Enfermagem ao paciente psiquiátrico e ao paciente de outras especialidades: percepção da Enfermagem. REME - Rev Min Enferm. 2019.

23. OLIVEIRA FV. Animalidade, loucura e biopolítica em Foucault. Griot: Revista de Filosofia, 2020; 20(3): $154-163$.

24. PAULA GS, et al. Violência relacionada ao trabalho na psiquiatria: percepção dos trabalhadores de enfermagem. SMAD, Rev. Eletrônica Saúde Mental Álcool Drog. (Ed. port.), 2017; 13(2): 86-92.

25. PROVIDELLO GGD, YASUI S. A loucura em Foucault: arte e loucura, loucura e desrazão. História, Ciências, Saúde - Manguinhos, 2013; 20(4): 1515-1529.

26. SAMPAIO ML, BISPO JÚNIOR JP. Entre o enclausuramento e a desinstitucionalização: a trajetória da saúde mental no Brasil. Trabalho, Educação e Saúde, 2021; 19: e00313145.

27. SANTOS CSCS, et al. Evaluation of work overload in the nursing team and the impact on the quality of care. Research, Society and Development, 2020; 9(5): e94953201.

28. SCOZZAFAVE MCS, et al. Riscos psicossociais relacionados ao enfermeiro no hospital psiquiátrico e estratégias de gerenciamento. Rev. Bras. Enfermagem, 2019; 72(4): 834-840.

29. SILVA CC, et al. Perfil dos profissionais de enfermagem portadores da síndrome de Burnout: uma revisão integrativa de literatura. Braz. J. Hea. Rev., 2020; 3(3): 4965-4979.

30. SOUSA KHJF, et al. Risks of illness in the work of the nursing team in a psychiatric hospital. Rev. Latino-Am. Enfermagem, 2018; 26: e3032.

31. TREVISAN E, et al. Satisfaction and work overload at Psychosocial Care Centers - Alcohol and Drugs in the Minas Triangle region, Brazil. Rev Bras Med Trab., 2019; 17(4): 511-520.

32. VIEIRA GLC. Satisfação e sobrecarga de trabalho entre técnicos de enfermagem de hospitais psiquiátricos. Revista Portuguesa de Enfermagem de Saúde Mental, 2017; 17: 43-49.

33. WORLD HEALTH ORGANIZATION (WHO). WHO-SATIS consumer's and caregivers' satisfaction with mental health services a multisite study. Geneva: Mental Health World Organization, 1996.

34. ZHENG Z, et al. Job satisfaction and resilience in psychiatric nurses: A study at the Institute of Mental Health, Singapore. Int J Ment Health Nurs., 2017; 26(6): 612-619. 